$$
\begin{aligned}
\Gamma & =\frac{16 h^{3}\left(c_{11}-c_{12}\right)}{3} \frac{\partial^{2}}{\partial \bar{z}^{2}}(\phi+i \psi), \\
\Psi_{0} & =\frac{10 h c_{44}}{3} \frac{\partial}{\partial \bar{z}}\left(w^{*}+2 \phi+2 i \psi\right), \\
D^{*} & =4 \frac{\partial}{\partial \bar{z}}(\phi+i \psi) .
\end{aligned}
$$

The function $\psi$ satisfies the equation

$$
\psi-\frac{c_{11}-c_{12}}{c_{44}} \cdot \frac{h^{2}}{5} \nabla_{1}^{2} \psi=0 .
$$

\title{
SOME REMARKS ON THE FLAT PLATE BOUNDARY LAYER*
}

\section{By J. A. LEWIS AND G. F. CARRIER (Brown University)}

1. Introduction. In a recent paper ${ }^{1}$, the Blasius solution for the boundary layer flow past a flat plate was supplemented by an investigation of the character of the flow field near the leading edge of the plate. In that paper, the leading edge solution (of the Stokes type) was matched numerically to the Blasius solution. At first glance, therefore, it would seem desirable to find the solution of the Oseen type and either verify or improve this match. In the present paper we shall compute the exact solution of the Oseen equations associated with the flow past a semi-infinite plate and shall present arguments which verify our belief that this solution is physically not acceptable. However, we shall introduce a modification of the Oseen linearization such that the exact solution of the modified equations completes the flow pattern with a reasonable degree of accuracy.

We shall also indicate an iteration procedure from which the exact flow pattern (i.e., the exact solution of the non-linear equations) can be obtained as the limit of a rapidly converging sequence of functions. Since the actual calculation associated with each step of this iteration would be very tedious, and since the solution given by Blasius ${ }^{1}$ and the present result give the most interesting information, we shall not complete the integrations.

Finally, we shall indicate the "modified Oseen solution" for the flow past a flat plate of finite length. Again, the interest does not seem to justify the necessary algebra so that no numerical results are obtained.

2. The Oseen linearization. The equations which govern the flow of a viscous, incompressible fluid

$$
\begin{gathered}
u_{i} \frac{\partial u_{i}}{\partial x_{i}}+\rho^{-1} \frac{\partial p}{\partial x_{i}}=\nu \frac{\partial^{2} u_{i}}{\partial x_{i} \partial x_{i}} \\
\frac{\partial u_{i}}{\partial x_{i}}=0
\end{gathered}
$$

*Received Nov. 5, 1948.

${ }^{1}$ G. F. Carrier and C. C. Lin, On the nature of the boundary layer near the leading edge of a flat plate, Q. Appl. Math. 6, 63-68 (1948). 
can be linearized in a manner first suggested by Oseen ${ }^{2}$. For our problem, the arguments leading to such a linearization might be the following. The stream function and velocity functions defining the flow must have a branch point at the leading edge of the plate (i.e., the origin) and, furthermore, the velocities vanish at this point. This implies that the third term in Eq. (1) will provide much larger contributions near the origin than will the first term. Moreover, the velocity at large distances from the plate is essentially the constant free stream velocity, $u_{0}$. Thus, one replaces Eq. (1) by

$$
u_{0} \frac{\partial u_{i}}{\partial x_{1}}+\rho^{-1} \frac{\partial p}{\partial x_{i}}=\nu \frac{\partial^{2} u_{i}}{\partial x_{i} \partial x_{i}}
$$

and anticipates that the solution of Eq. (3) will demonstrate the proper qualitative behavior very near the plate and very far from the plate. However, if the very low speed field is separated from the "far field" by a region in which the non-linear terms predominate, we shall be faced with a phenomenon not unlike the Stokes phenomenon encountered when investigating asymptotic solutions of ordinary differential equations. In fact, we cannot anticipate that the solution of Eqs. (2) and (3), which holds for the far field, will be the same solution which holds in the slow flow region even though in each region the asymptotic form of the flow functions must be solutions of these equations. Nevertheless, it is profitable to find the solution of these Oseen equations and then utilize the extra information at our disposal to modify them and render the theory more applicable to our problem. Therefore, let us define $u_{1}=u_{0}\left[1+\psi_{y}\right], u_{2}=-u_{0} \psi_{x}$, $x=u_{0} x_{1} / \nu, y=u_{0} x_{2} / \nu$, where $x_{1}, x_{2}, u_{1}, u_{2}$ are the physical coordinates and the associated velocity components. With these definitions, Eqs. (2) and (3) become

$$
\Delta\left(\Delta-\frac{\partial}{\partial x}\right) \psi=0
$$

where $\Delta$ is the Laplacian operator. The boundary conditions require that $\psi_{x}, \psi_{y} \rightarrow 0$ far from the plate and that $\psi_{x}=0, \psi_{y}=-1$ on the plate, (i.e., on $y=0, x>0$ ). We note here that $\psi, \psi_{y}, \psi_{y y}$ are continuous across the plate but that $\psi_{y y}$ will be discontinuous. Indeed, let us define $f(x)$ to be the discontinuity in $\psi_{y y}$ across the plate and note that $f(x)$ vanishes when $x<0$ and that $f$ is twice the velocity gradient $\left(\partial u_{1} / \partial x_{2}\right.$ in dimensionless form) along the plate. We may most readily formulate the problem by the use of Fourier transforms. In fact, we define

$$
\bar{\psi}(\xi, \eta)=\int_{-\infty}^{\infty} \psi(x, y) e^{-i(\xi x+\eta y)} d x d y
$$

multiply Eq. (4) by exp $[-i(\xi x+\eta y)]$, and integrate over the infinite region. Under our boundary conditions, integration by parts with respect to $y$ leads to

$$
\int_{-\infty}^{\infty} \int_{-\infty}^{\infty}\left(\frac{\partial^{2}}{\partial x^{2}}-\eta^{2}\right)\left(\frac{\partial^{2}}{\partial x^{2}}-\frac{\partial}{\partial x}-\eta^{2}\right) \psi(x, y) e^{-i(\xi x+\eta \nu)} d x d y=\int_{-\infty}^{\infty}-i \eta f(x) e^{-i \xi x} d x,
$$

where $f(x)$ is defined in the foregoing. Defining $\bar{f}(\xi)$ in the obvious manner and completing the integration, we obtain

$$
\left(\xi^{2}+\eta^{2}\right)\left(\xi^{2}+\eta^{2}+i \xi\right) \bar{\psi}=-i \eta \bar{f} .
$$

${ }^{2}$ H. Lamb, Hydrodynamics, Dover Publications, New York, 1945, p. 610. 
At this stage, we can avoid future difficulties by observing that Eq. (7) is the limit (as $k \rightarrow 0$ ) of the equation

$$
\left(\xi^{2}+\eta^{2}+k^{2}\right)\left(\eta^{2}+[\xi+i][\xi-i k]\right) \bar{\psi}=-i \eta \bar{f} .
$$

We also note that the boundary conditions $\psi_{\nu}=-1$ on the plate may be written as the limit, as $\alpha \rightarrow 0$, of $\psi_{y}(x, 0)=-e^{-\alpha x}$ on $x>0$. Therefore, we now are prepared to solve Eq. (8) for $\bar{\psi}$ and $f$ according to the boundary conditions, with the obvious intent of carrying out the indicated limiting processes. Let us now define the function $\bar{\varphi}(\xi, y)$ to be

$$
\begin{aligned}
\bar{\varphi}(\xi, y) & =\frac{1}{2 \pi} \int_{-\infty}^{\infty} \bar{\psi}(\xi, \eta) e^{i \eta \nu} d \eta \\
& =\int_{-\infty}^{\infty} \psi(x, y) e^{-i \xi x} d x .
\end{aligned}
$$

The latter identity follows from the conventional inversion formula. Solving Eq. (8) for $\bar{\psi}$ and performing the integration in Eq. (9) by the use of simple contour integrals, we obtain

$$
\bar{\varphi}(\xi, y)=\frac{\bar{f}\left[\exp \left\{-|y|\left(\xi^{2}+k^{2}\right)^{1 / 2}\right\}-\exp \left\{-|y|(\xi+i)^{1 / 2}(\xi-i k)^{1 / 2}\right\}\right]}{2 i(1-k)(\xi-i k)}
$$

and, in particular,

$$
\bar{\varphi}_{y}(\xi, 0)=\frac{\bar{f}(\xi)}{2(\xi-i k)^{1 / 2}\left[(\xi+i k)^{1 / 2}+(\xi+i)^{1 / 2}\right]} .
$$

However, our boundary conditions are associated with $\bar{\varphi}_{\nu}$, and it is convenient to define

$$
\bar{\varphi}_{\nu}(\xi, 0)=\bar{u}_{0}(\xi)+\bar{u}_{1}(\xi)
$$

where

$$
\vec{u}_{0}(\xi)=-\int_{0}^{\infty} e^{-\alpha x} e^{-i \xi x} d x=\frac{i}{\xi-i \alpha}
$$

and

$$
\bar{u}_{1}(\xi)=\int_{-\infty}^{0} u(x) e^{-i \xi x} d x ;
$$

that is, $\bar{u}_{0}$ is the transform of $\psi_{y}$ along the plate and $\bar{u}_{1}$, of $\psi_{y}$ along the line $y=0$ $x<0$. The latter function is, of course, not known. Using these substitutions in Eq. (11)

$$
\bar{u}_{1}(\xi)+i /(\xi-i \alpha)=\frac{\bar{f}(\xi)}{2(\xi-i k)^{1 / 2}\left[(\xi+i k)^{1 / 2}+(\xi+i)^{1 / 2}\right]} .
$$

We can now determine $\bar{f}$ and $\bar{u}_{1}$ by the Wiener-Hopf method. ${ }^{3}$ We note that, by definition, $\bar{u}_{1}(\xi)$ is an analytic function of the complex variable $\xi$ in some upper halfplane which includes the real axis, provided we anticipate that $\psi_{y}$ should decay ex-

${ }^{3}$ This technique has been extensively used, of late, on diffraction problems by $H$. Levine, J. Schwinger, A. E. Heins, and others. 
ponentially in $(-x)$ on the negative $x$-axis. Similarly, $\bar{f}(\xi)$ is analytic in some lower half-plane including the real axis. Therefore, when we rewrite Eq. (14) in the form

$$
\begin{gathered}
\bar{u}_{1}(\xi)\left[(\xi+i k)^{1 / 2}+(\xi+i)^{1 / 2}\right]+\frac{i}{\xi-i \alpha}\left\{\left[(\xi+i k)^{1 / 2}+(\xi+i)^{1 / 2}\right]\right. \\
\left.-(i)^{1 / 2}\left[(\alpha+k)^{1 / 2}+(\alpha+1)^{1 / 2}\right]\right\}=\frac{1}{2} \bar{f}(\xi)(\xi-i k)^{-1 / 2} \\
-\frac{i}{\xi-i \alpha}(i)^{1 / 2}\left[(\alpha+k)^{1 / 2}+(\alpha+1)^{1 / 2}\right]
\end{gathered}
$$

the left-hand side is analytic in the upper half-plane mentioned before and the right side is analytic in the lower half-plane. Thus, by the arguments of analytic continuation, each side is equal to a constant, which is readily seen to be zero. Consequently, in the limit $k \rightarrow 0, \alpha \rightarrow 0$

$$
\bar{f}(\xi)=2 i\left(\frac{i}{\xi}\right)^{1 / 2}
$$

and we find by the conventional inversion formula that

$$
f(x)=2(\pi x)^{-1 / 2} \text {. }
$$

But $f(x)$ is twice the velocity gradient along the plate. Thus we obtain $.555 / x^{1 / 2}$ for the velocity gradient, as compared with $.332 / x^{1 / 2}$ which, of course, is the Blasius result ${ }^{1}$. Thus, we must conclude that the region anticipated earlier in this section exists and that the solution is not valid.

3. The modified Oseen solution. It is most convenient to introduce our proposed modification of the Oseen linearization with a minimum of a priori argument and, having found the desired solution, to establish justification arguments using the result to strengthen these arguments. We therefore merely suppose, for the moment, that the inertia term of Eq. (1) should be replaced, not by $u_{0} \partial u_{i} / \partial x_{1}$, but by $u^{*} \partial u_{i} / \partial x_{1}$, where $u^{*}$ is some weighted average of the horizontal velocity component. It is evident that different methods of averaging would give different $u^{*}$ values so we may write $u^{*}=c u_{0}$ where we expect that $0<c<1$. Reserving the privilege of choosing $c$ after the equations are solved, we replace $u_{0}$ by $c u_{0}$ in Eq. (3), and Eq. (4) becomes

$$
\Delta\left(\Delta-c \frac{\partial}{\partial x}\right) \psi=0 \text {. }
$$

It is evident that the solution can be found as before, and in fact, we now obtain

$$
f(c, x)=2(c / \pi x)^{1 / 2}
$$

where $f$ is again the jump in $\psi_{y y}$ across the plate.

It can also be shown after some manipulation that

$$
\psi \equiv \frac{1}{2 \pi} \int_{-\infty}^{\infty} \bar{\varphi}(\xi, y) e^{i \xi x} d \xi
$$

can be written

$$
\psi=\int_{-\infty}^{x}\left[(r+x) / 2 c \pi r^{2}\right]^{1 / 2}\left(1-e^{e(x-r) / 2}\right) d x
$$


and that, in particular

$$
\begin{gathered}
v^{\prime}=-\psi_{x}=-\left[(r+x) / 2 c \pi r^{2}\right]^{1 / 2}\left(1-e^{c(x-r) / 2}\right) \\
u^{\prime}=\psi_{y}=\int_{-\infty}^{x} \frac{\partial}{\partial y}\left\{\left[(r+x) / 2 c \pi r^{2}\right]^{1 / 2}\left(1-e^{c(x-r) / 2}\right)\right\} d x
\end{gathered}
$$

where $r^{2}=x^{2}+y^{2}$.

We now observe that the velocity gradient at the plate which is associated with the solution agrees with the Blasius result when $c$ is chosen to be about .35. We also observe that the character of the far field flow is unaffected by changes in $c$ provided $c>0$. That is, the leading terms in the dimensionless velocities $u^{\prime}$ and $v^{\prime}$ are essentially independent of $c$ for large distances from the plate. Thus the following observations are valid: the modified Oseen equation (18) is as valid as Eq. (4) for predicting the far field behavior and the Stokes (slow) flow field for any positive $c$. The choice $c=.35$ allows us to use a single solution of Eq. (18) to predict these asymptotic flows and also to approximate the flow in the intermediate region. Since any Oseen attack is intended to do no more than this, it appears that this modification constitutes an improvement (for this particular problem) over the conventional attack. It must be noted, however, that we needed to know the value of $\psi_{y y}$ at the plate in order to choose our correction.

There are various ways in which one might formulate a criterion for choosing $c$ when the velocity gradient at the obstacle is not known. We might, for example, demand that the average (along the negative real axis) of the neglected inertia term be made to vanish, that is, that

$$
\int_{-\infty}^{0}\left(1+u^{\prime}-c\right) \frac{\partial u^{\prime}}{\partial x} d x=0 .
$$

This, in fact, yields $c=1 / 2$. A second way would be to weight this average. For example, if we wished to make the inertia term have mean value zero in a wedge shaped region ahead of the plate, we could require that

$$
\int_{-0}^{0} x\left(1+u^{\prime}-c\right) \frac{\partial u^{\prime}}{\partial x} d x=0 .
$$

Unfortunately, however, this integral does not exist, even in the Abelian sense. However, using the argument that when $u^{\prime}$ is very small the value of $c$ does not matter, we might weight the inertia term with $u^{\prime}$ itself. That is, we might require that

$$
\int_{-\infty}^{0} u^{\prime}(x, 0)\left[1+u^{\prime}-c\right] \frac{\partial u^{\prime}}{\partial x} d x=0 .
$$

This produces the result $c=1 / 3$ which is, of course, an excellent approximation to the value formerly found. Since this averaging makes sense physically and yields the proper result in at least one case, we suggest that, in general, the Oseen linearization associated with the viscous flow past an obstacle can be improved by using $u^{*}$ instead of $u_{0}$, where $u^{*}$ is found by the foregoing averaging process. However, in view of the fact that this yields $u^{*}=c u_{0}=u_{0} / 3$ without regard to the form of $u^{\prime}$ (and therejore without dependence on the symmetric obstacle shape), one must immediately conclude that $u^{*} \simeq u_{0} / 3$ for any symmetric obstacle. The flow so obtained can then be expected to be valid in the Stokes slow flow region near the obstacle, far from the obstacle, and 
in some strip along $x<0,|y|<y_{0}$. This is certainly as large a region of validity as one could expect from such an asymptotic solution.

4. The exact solution. If we now write down the exact equation for the stream function $\psi$ (as defined before) we obtain

$$
\Delta\left(\Delta-c \frac{\partial}{\partial x}\right) \psi=F(\psi)
$$

where $F(\psi)$ is that portion of the inertia term not contained in $c \psi_{s}$. We can now find a Green's function for this equation (treating $F$ as a non-homogeneous term) and write

$$
\psi=\psi_{0}(x, y)+\iint_{-\infty}^{\infty} G\left(x, x_{0} ; y, y_{0}\right) F\left(\psi\left[x_{0}, y_{0}\right]\right) d x_{0} d y_{0}
$$

where $\psi_{0}$ is the modified Oseen solution just obtained. We note that if a function $\psi^{*}$, which was very nearly the exact solution of Eq. (24), were placed in the integral term, the function $\psi$ obtained from the integration and addition of $\psi_{0}$ would, in general, be still more accurate. This, of course, is a standard procedure. However, we have at our disposal the fact that for $|\operatorname{arc} \tan (y /|x|)|<$ (say) $\pi / 18$, the Blasius solution is very accurate, and for the remaining field the present solution seems adequate. Therefore, if one divided the integral of Eq. (25) into two parts, chose $\psi^{*}$ as suggested here, and carried out the integration, he could expect a marked improvement in the result. Actually, the results already in hand seem good enough so that the amount of labor required is essentially prohibitive. Thus we shall not pursue the question further except to write down the appropriate Green's function. When $\psi^{*}$ is odd in $y$, the integral of Eq. (25) will always obey the conditions implying $\psi=\psi_{v}=0$ on $y=0, x>0$, if the Green's function is

$$
\begin{aligned}
G_{0}=\operatorname{Re} & \iint_{-\infty}^{\infty} \ln \left[\left(z^{1 / 2}-z_{1}^{1 / 2}\right)\left(z^{1 / 2}-\bar{z}_{1}^{1 / 2}\right)\right] e^{c\left(x_{1}-x_{0}\right) / 2} \\
& \cdot K_{0}\left\{\frac{c}{2}\left[\left(x_{1}-x_{0}\right)^{2}+\left(y_{1}-y_{0}\right)^{2}\right]^{1 / 2}\right\} d x_{1} d y_{1},
\end{aligned}
$$

where $z=x+i y ; 0<\arg z^{1 / 2}<\pi ; \pi<\arg (\bar{z})^{1 / 2}<2 \pi ;$ and $K_{0}$ is the modified Hankel function of order zero.

5. The plate of finite length. The technique by which the foregoing problem was solved cannot be directly utilized when the plate is of finite length. However, if we let the plate extend over the interval $0<x<A$, we may write (for the jump in $\psi_{v y}$ )

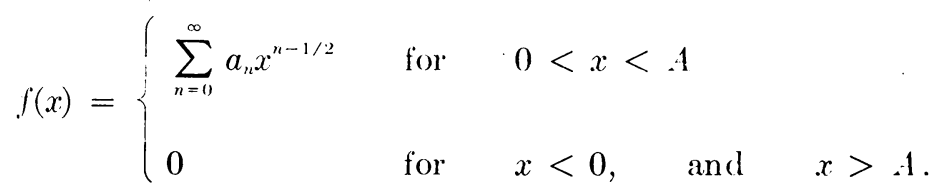

When this is done, $\psi_{y}(x, 0)$ in $0<x<A$ can be written

$$
\psi_{y}(x, 0)=\sum_{n} a_{n}^{\prime} x^{n} \exp \left(k_{n} x\right)
$$


It can also readily be shown that one may successively find the $a_{n}^{\prime}$ (and hence $a_{n}$ ) so that

$$
\psi_{y}(x, 0)=-1 \quad \text { on } \quad 0<x<A .
$$

The series will fail to converge, of course, at $x=A$. However, $a_{0}$ can readily be shown to exceed 1.11 (the value obtained for the semi-infinite plate) and thus the finite plate consideration does not make the unmodified Oseen procedure more valid. In fact, one might use the modified procedure to improve the finite plate solution although, for this problem, we do not know the velocity gradient at the plate as we did for the long plate. In any event, the numerical evaluation of the velocity field will not be pursued here in any detail.

\title{
AN ITERATIVE METHOD FOR FINDING THE SMALLEST EIGENVALUE OF A MATRIX*
}

\author{
By JOHN B. LOHMAN (Brown University)
}

The eigenvalues and eigenvectors of a matrix $A$ are often found by repeated premultiplication of an arbitrary column vector $v$ by the matrix $A$. The products $A^{n} v$ converge to the eigenvector $v_{1}$ corresponding to the eigenvalue $\lambda_{1}$ largest in absolute magnitude; convergence is rapid if $\lambda_{1}$ is much larger than any other eigenvalue of $A$.

If one already has approximate values of the eigenvalues of $A$, this iterative method can be modified to give more rapid convergence. Aitken ${ }^{1}$ suggests subtracting a scalar matrix $a I$ ( $I$ denotes the unit matrix) from $A ; a$ is chosen so as to give the maximum separation of $\lambda_{1}$. Kincaid ${ }^{2}$ carries out the iteration using a polynomial $P(A)$ in $A$, in place of $A$ itself; $P(A)$ is so chosen that the eigenvalues not immediately sought are as nearly zero as possible.

Iteration for the smallest eigenvalue of a matrix $A$ is commonly performed using the inverse matrix $A^{-1}$. This note deseribes a modified procedure leading to more rapid convergence.

Let the eigenvalues of $A$ be $.22, .4,1,2$, and assume we know them only to within $10 \%$. Simple inversion of $A$ yields $A^{-1}$ with eigenvalues $4.5,2.5,1, .5$; the largest eigenvalue of $A^{-1}$ (corresponding to the smallest eigenvalue of $A$ ) is 1.8 times as large as the second-largest. Assume we believe the smallest eigenvalue of $A$ to be .2 instead of .22; subtract the scalar matrix .2I from $A$, and then invert; the resulting matrix $(A-.2 \mathrm{I})^{-1}$ has eigenvalues $50,5,1.25, .56$. But we can still improve the separation by subtracting another scalar matrix. We choose the mean of the second largest and the smallest eigenvalues (a different choice would be appropriate if some eigenvalues were negative), for maximum separation of the largest and the second largest eigenvalues. Our final modified matrix, $(A-.2 \mathrm{I})^{-1}-2.78 \mathrm{I}$, has eigenvalues $47.22,2.22,-1.53,-2.22$. The ratio of largest to second largest eigenvalue has been increased from 1.8 to 21.3.

Since the modified matrix has all but one of its eigenvalues tightly clustered about zero, it is not a suitable form from which to determine these other eigenvalues.

${ }^{*}$ Received July 22, 1948. This work was supported under Navy contract.

${ }^{1}$ A. C. Aitken, Proc. Roy. Soc. Edinburgh 57, 269-270 (1937).

${ }^{2}$ W. M. Kincaid, Q. Appl. Math. 5, 320-345 (1947). 